\title{
THE ICHTHYOFAUNA OF THE BRAZILIAN SURF ZONE: A COMPILATION FOR ECOLOGICAL COMPREHENSION PER REGION
}

\author{
Fábio Magno da Silva SANTANA ${ }^{1,2,4}$ \\ William SEVERI ${ }^{3}$ \\ Flavo Elano Soares de SOUZA ${ }^{4}$ \\ Maria Elisabeth de ARAÚJO²
}

Recebido em: 02/02/2013

Aceito em: $25 / 05 / 2013$

\section{ABSTRACT}

The ichthyofauna of the surf zones of the Brazilian coast was determined and characterized by the territorial division of Brazil. We compiled 22 scientific papers published in scientific journals which presented the distribution of fish species in these sites. Two hundred and thirty-nine species belonging to seventy-one families of fish were validated and listed. The northern Brazil showed no study in this habitat. The southeast region showed a higher richness of species, families and uniqueness of species, whereas the southern had lower values of richness among the regions studied. In the northeast, most of the unique

species are associated with reef environments and the southern presented species typical in freshwater among the unique ones. Finally, the presence of 239 fish species and 71 families represents a considerable number for the ichthyofauna of the Brazilian coast surf zone, for this environment has been scarcely studied. However, because there have been found species inhabiting other environments and by the presence of 61 species with $100 \%$ frequency of occurrence in the three regions studied, it can be concluded that it is an ecologically important site.

Key words: fish, sea beaches, trawls, Brazil, bibliography

\section{RESUMO}

A ictiofauna das zonas de arrebentação da costa brasileira foi determinada e caracterizada através da divisão territorial do Brasil. Foram compilados 22 artigos científicos publicados em revistas científicas indexadas que apresentavam distribuição de espécies de peixes nestes locais. Foram listadas e validadas 239 espécies pertencentes a 71 famílias de peixes. A região norte do Brasil não apresentou estudo neste habitat. A região sudeste teve maior riqueza de espécies, famílias e exclusividade de espécies, enquanto que a região sul apresentou menores valores de riqueza entre as regiões estudadas. No nordeste, a maioria das exclusivas tem associação com ambientes recifais e a região sul apresentou, dentre as exclusivas, espécies típicas de água doce. Finalmente, a presença de 239 espécies de peixes e 71 famílias representa um número considerável para a ictiofauna da zona de arrebentação do litoral brasileiro, pois esse ambiente ainda é pouco estudado. Contudo, por serem encontradas espécies que habitam outros ambientes e pela presença de 61 espécies com 100\% de frequência de ocorrência nas três regiões estudadas, pode-se concluir que seja um local ecologicamente importante.

Palavras-chave: peixes, praias marinhas, redes de arrasto, Brasil, levantamento bibliográfico.

\section{INTRODUCTION}

Brazil has a coastline with an approximate length of 9,200 km (KNOPPERS et al., 2002), adding to the recesses, being the most extensive inter and subtropical coast in the world (AB'SÁBER, 2003). It is characterized by several ecosystems, including estuaries, coral reefs,

\footnotetext{
${ }^{1}$ Doutorando do Programa de Pós-Graduação em Oceanografia da Universidade Federal de Pernambuco. Email: fabiomagnos@yahoo.com.br

${ }^{2}$ Laboratório de Ictiologia Marinha Tropical, Departamento de Oceanografia, Universidade Federal de Pernambuco. Email: betharau08@gmail.com ${ }^{3}$ Laboratório de Ictiologia, Departamento de Pesca e Aquicultura, Universidade Federal Rural de Pernambuco. Email: wseveri@depaq.ufrpe.br

${ }^{4}$ Escola Agrícola de Jundiaí, Unidade Acadêmica Especializada em Ciências Agrárias, Universidade Federal do Rio Grande do Norte. Email: flavogeo@gmail.com
} 
SANTANA, Fábio M. da S. et. al. The ichthyofauna of the brazilian surf zone: a compilation for ecological comprehension per region.

lagoons, bays and surf zones, inhabited by many marine organisms that represent important natural resources (GURGEL et al., 2012).

Along the entire coast, there are surf zones which, by definition, are an integral part of the fore shore, that extends from the first line of breaking waves to the lower limit of the beach face (VELOSO and NEVES, 2009). Many species of fish live in them, especially juveniles, who use them as nursery (ROBERTSON and LENANTON, 1984; GODEFROID et al., 2001) as well as area for food and protection from predators (BLABER and BLABER, 1980; LASIAK, 1984a, b; WHITFIELD, 1996). A large number of species are found in the surf zone, although it is dominated by a few (GIANNINI and PAIVA FILHO, 1995; FELIX et al., 2007a; SANTANA and SEVERI, 2009). The dynamics of the movements of waves and sudden variations in environmental conditions cause changes in the behavior of communities that use the surf zone, turning these places into interesting sites for studies (WRIGHT and SHORT, 1984) on ecosystem interconnection.

Researches on composition and structure of fish communities in surf zones are scarce if compared to other coastal environments, such as estuaries and reefs. Although most of these works concern the beaches located in the southern and southeastern Brazil (ARAÚJO et al., 2008), the number of studies has grown in the northeast (e.g. OLIVEIRA-SILVA, PESO-AGUIAR and LOPES, 2008; LIRA and TEIXEIRA, 2008, SANTANA and SEVERI op cite; GURGEL op cite). However, there is no work that presents the composition of the fish assemblage of the surf zone for the entire Brazilian coast or that compares these assemblies by territorial regions.

Compilations of data on fauna are important to help understand the geographic distribution and the species' macro ecology traits (ARAÚJO and FEITOSA, 2003; BARLETTA and BLABER, 2007). Data can be gathered as a list of an enclosed environment, including comments about geographic distribution (PASSOS, 2012).

The objective of this study is to collect data to generate a list of the ichthyofauna of the Brazilian coast surf and infer the main ecological differences in the structure of the fish community in the regions analyzed.

\section{MATERIAL AND METHODS}

The criterion used to select the area covered by this study was based on the availability of scientific papers published in indexed journals that would approach the composition of fish communities in surf zones in any Brazilian locality (Tab. 1). The figure 1 ilustred the map of Brazil indicating the states where data were compiled in this study. In order to validate the taxonomy it was carried out a review of the scientific names of species according to Menezes et al. (2003), Araújo, Teixeira and Oliveira (2004) and Froese and Pauly (2008), aiming to update the names considered inappropriate or synonymic.

The frequency of occurrence (FO) of the species was determined by the presence or absence of each species per study area analyzed (Tab. 1). Each territorial region was compared for obtaining these relative frequencies for Brazil. 


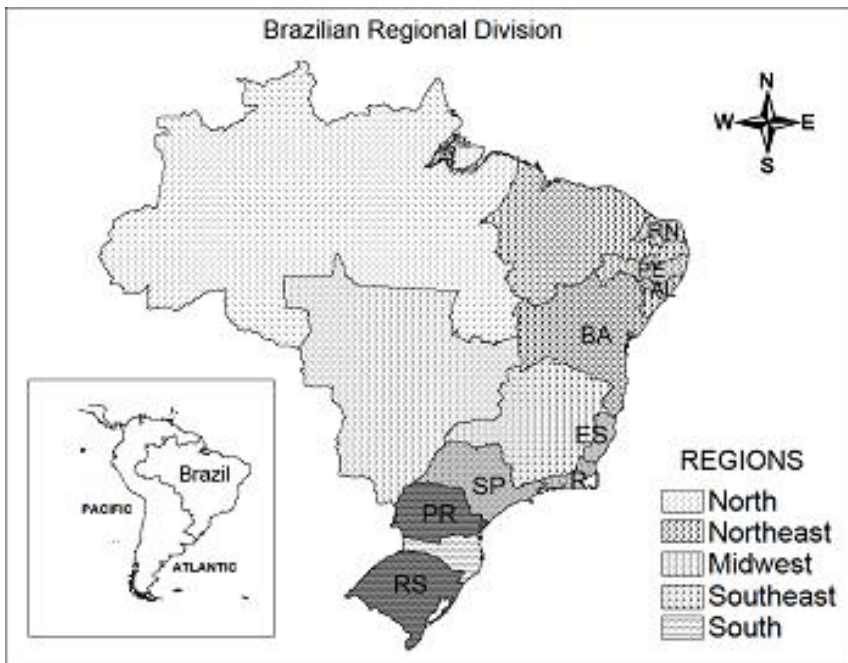

Figure 1 - Map of Brazil indicating the states where data were compiled in this study. $\mathrm{RN}=$ Rio Grande do Norte, $\mathrm{PE}=$ Pernambuco, $\mathrm{AL}=$ Alagoas, $\mathrm{BA}=\mathrm{Bahia}, \mathrm{ES}=$ Espírito Santo, RJ= Rio de Janeiro, $\mathrm{SP}=\mathrm{São}$ Paulo, PR= Paraná e RS= Rio Grande do Sul.

Table 1 - List of scientific papers used to obtain the ichthyofauna data of the Brazilian surf zone.

\begin{tabular}{|c|c|c|c|c|}
\hline Region & State & City & Coordinates & References \\
\hline Northeast & Alagoas & Maceió & No Informed & $\begin{array}{l}\text { Teixeira and Almeida, } \\
1998\end{array}$ \\
\hline Northeast & Bahia & Saubara & $12^{\circ} 47^{\prime} \mathrm{S} ; 038^{\circ} 46^{\prime} \mathrm{W}$ & $\begin{array}{l}\text { Oliveira-Silva et al., } \\
2008\end{array}$ \\
\hline Northeast & Pernambuco & Itamaracá & $07^{\circ} 43,722^{\prime} \mathrm{S} ; 034^{\circ} 49,536^{\prime} \mathrm{W}$ & Lira and Teixeira, 2008 \\
\hline Northeast & Pernambuco & Itamaracá & $\begin{array}{l}07^{\circ} 43^{\prime} 42,9^{\prime \prime} \mathrm{S} \\
034^{\circ} 49^{\prime} 32,1^{\prime \prime} \mathrm{W}\end{array}$ & $\begin{array}{l}\text { Santana and Severi, } \\
2009\end{array}$ \\
\hline Northeast & $\begin{array}{l}\text { Rio Grande do } \\
\text { Norte }\end{array}$ & Natal & $\begin{array}{l}05^{\circ} 52^{\prime} 4730^{\prime \prime} \mathrm{S} ; 35^{\circ} 10^{\prime} 6 \\
93^{\prime \prime} \mathrm{W}\end{array}$ & Gurgel et al., 2012 \\
\hline Southeast & Espírito Santo & Vitória & $20^{\circ} 18^{\prime} \mathrm{S} ; 40^{\circ} 17^{\prime} \mathrm{W}$ & Araujo et al, 2008 \\
\hline Southeast & Rio de Janeiro & Rio de Janeiro & $\begin{array}{l}22^{\circ} 54^{\prime}-23^{\circ} 04^{\prime} \mathrm{S} ; 43^{\circ} 34^{\prime}- \\
44^{\circ} 10^{\prime} \mathrm{W}\end{array}$ & $\begin{array}{l}\text { Pessanha and } \\
\text { Araújo,2003 }\end{array}$ \\
\hline Southeast & Rio de Janeiro & S. Francisco & $21^{\circ} 17^{\prime} \mathrm{S} ; 40^{\circ} 57^{\prime} \mathrm{W}$ & Gomes et al., 2003 \\
\hline Southeast & Rio de Janeiro & $\begin{array}{l}\text { Arraial do } \\
\text { Cabo/RJ }\end{array}$ & $22^{\circ} 58^{\prime} \mathrm{S} ; 42^{\circ} 01^{\prime} \mathrm{W}$ & Gaelzer et al.,2006 \\
\hline Southeast & Rio de Janeiro & Rio de Janeiro & No Informed & Vasconcellos et al., 2007 \\
\hline Southeast & Rio de Janeiro & Niterói & $22^{\circ} 53^{\prime} 14^{\prime \prime} \mathrm{S} ; 43^{\circ} 22^{\prime} 48^{\prime \prime} \mathrm{W}$ & $\begin{array}{l}\text { Monteiro-Neto et al., } \\
2008\end{array}$ \\
\hline Southeast & Rio de Janeiro & Arraial do Cabo & $22^{\circ} 58^{\prime} \mathrm{S} ; 42^{\circ} 01^{\prime} \mathrm{W}$ & $\begin{array}{l}\text { Gaelzer and Zalmon, } \\
2008\end{array}$ \\
\hline
\end{tabular}

${ }^{1}$ Doutorando do Programa de Pós-Graduação em Oceanografia da Universidade Federal de Pernambuco. Email: fabiomagnos@yahoo.com.br

${ }^{2}$ Laboratório de Ictiologia Marinha Tropical, Departamento de Oceanografia, Universidade Federal de Pernambuco. Email: betharau08@gmail.com ${ }^{3}$ Laboratório de Ictiologia, Departamento de Pesca e Aquicultura, Universidade Federal Rural de Pernambuco. Email: wseveri@depaq.ufrpe.br

${ }^{4}$ Escola Agrícola de Jundiaí, Unidade Acadêmica Especializada em Ciências Agrárias, Universidade Federal do Rio Grande do Norte. Email: flavogeo@gmail.com 
SANTANA, Fábio M. da S. et. al. The ichthyofauna of the brazilian surf zone: a compilation for ecological comprehension per region.

\begin{tabular}{|c|c|c|c|c|}
\hline Southeast & São Paulo & Guarujá & No Informed & $\begin{array}{l}\text { Paiva Filho and Toscano, } \\
1987\end{array}$ \\
\hline Southeast & São Paulo & South, North, & $23^{\circ}-25^{\circ} \mathrm{S} ; 45^{\circ}-49^{\circ} \mathrm{W}$ & Giannini and Paiva Filho, \\
\hline Southeast & São Paulo & & & \\
\hline Southeast & São Paulo & Ubatuba & $23^{\circ}$ e $25^{\circ} \mathrm{S} ; 45^{\circ}$ e $49^{\circ} \mathrm{W}$ & Gondolo et al., 2011 \\
\hline South & Paraná & $\begin{array}{l}\text { Pontal do } \\
\text { Paraná }\end{array}$ & No Informed & Godefroid et al., 1998 \\
\hline South & Paraná & $\begin{array}{l}\text { Pontal do } \\
\text { Paraná }\end{array}$ & No Informed & Godefroid et al., 2003 \\
\hline South & Paraná & $\begin{array}{l}\text { Pontal do } \\
\text { Paraná }\end{array}$ & No Informed & Felix et al., 2007(a) \\
\hline South & Paraná & $\begin{array}{l}\text { Pontal do } \\
\text { Paraná }\end{array}$ & $25^{\circ} 33^{\prime} 979^{\prime \prime S} 48^{\circ} 21^{\prime} 119^{\prime \prime} \mathrm{W}$ & Félix et al., 2007(b) \\
\hline South & Paraná & $\begin{array}{l}\text { Pontal do } \\
\text { Paraná }\end{array}$ & No Informed & $\begin{array}{l}\text { Félix-Hackradt et al., } \\
2010\end{array}$ \\
\hline South & $\begin{array}{l}\text { Rio Grande do } \\
\text { Sul }\end{array}$ & Rio Grande & No Informed & Lima and Vieira, 2009 \\
\hline South & $\begin{array}{l}\text { Rio Grande do } \\
\text { Sul }\end{array}$ & Rio Grande & No Informed & $\begin{array}{l}\text { Mont'Alverne et al., } \\
2012\end{array}$ \\
\hline
\end{tabular}

\section{RESULTS}

Two hundred and thirty nine species (Tab. 2) from 71 fish families were recorded. The 15 most specious families accounted for approximately $60 \%$ of all validated species (Fig. 2 ).

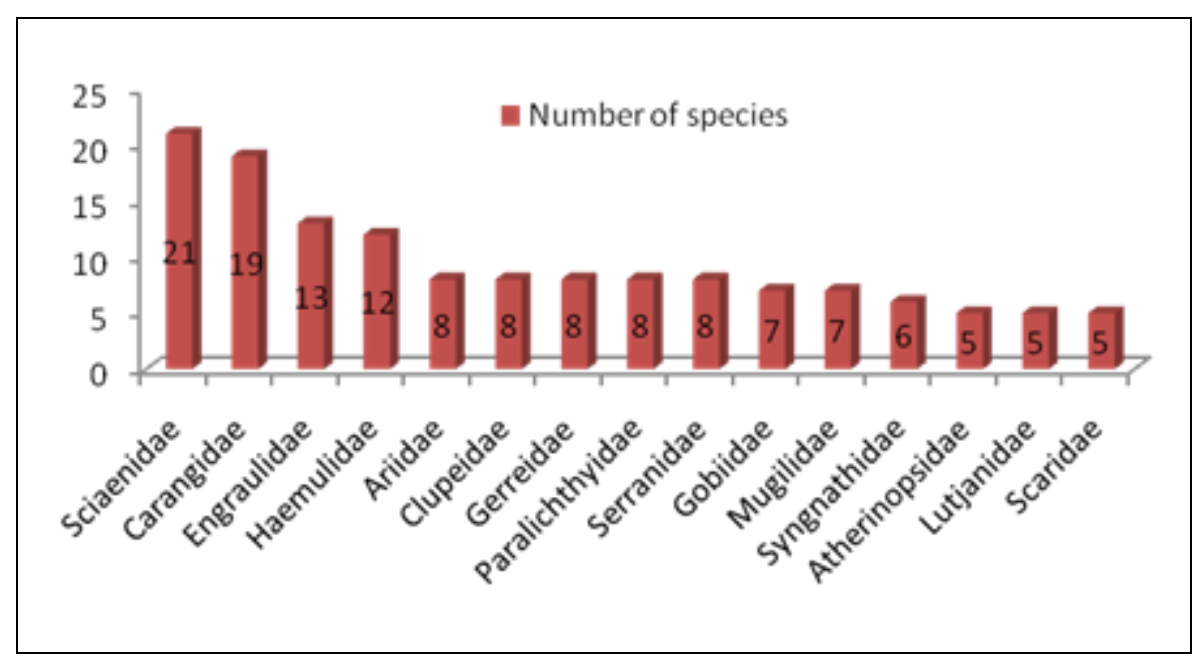

Figure 2 - Distribution of number of species per family in the surf zones of the Brazilian coast.

Regarding the occurrence of families and species analyzed by territorial region, the surf zones of southeast showed greater representativeness and southern lower. In terms of unique species, southeast (53) surpassed the northeast (45) and south (21) (Fig. 3). 


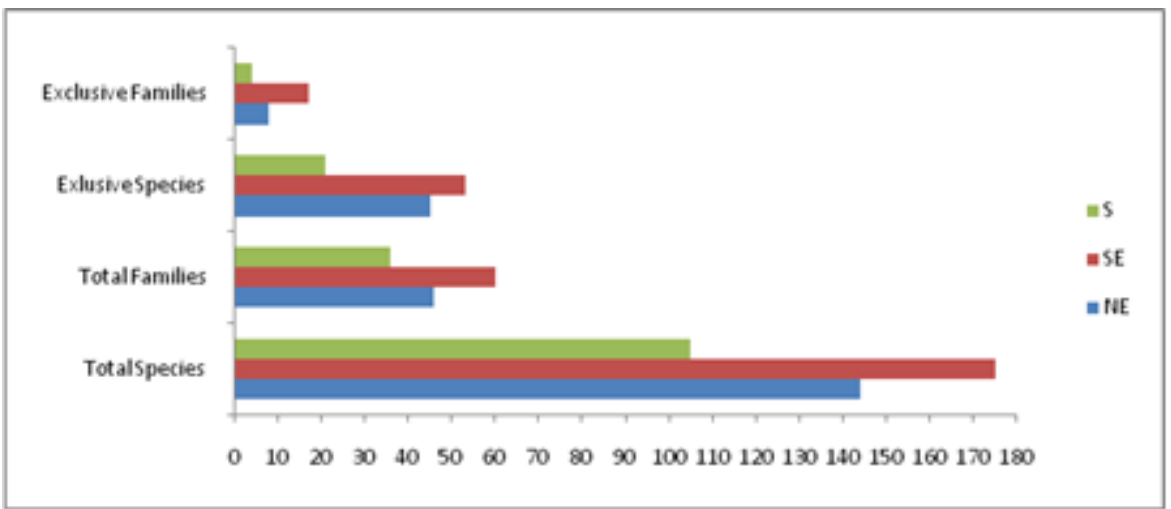

Figure 3 - Distribution of families and species of fish from the surf zone of the Brazilian coast by territorial region.

${ }^{1}$ Doutorando do Programa de Pós-Graduação em Oceanografia da Universidade Federal de Pernambuco. Email: fabiomagnos@yahoo.com.br

${ }^{2}$ Laboratório de Ictiologia Marinha Tropical, Departamento de Oceanografia, Universidade Federal de Pernambuco. Email: betharau08@gmail.com ${ }^{3}$ Laboratório de Ictiologia, Departamento de Pesca e Aquicultura, Universidade Federal Rural de Pernambuco. Email: wseveri@depaq.ufrpe.br 
SANTANA, Fábio M. da S. The ichthyofauna of the brazilian surf zone: a compilation for ecological comprehension per region.

Table 2 - List of species from surf zone of the Brazilian coast with their valid nomenclature and percentage of frequency of occurrence (FO\%). northeast (NE), exclusive occurrence in the northeast surfzone (ENE), southeast (SE), exclusive occurrence in the southeast surfzone (ESE), south (S), exclusive occurrence in the south surfzone (ES). The species common to all regions are in bold. The $x$ accuses the uniqueness of the species in the region.

\begin{tabular}{|c|c|c|c|c|c|c|c|c|c|}
\hline Valid Nomenclature & Cited Nomenclature & & $\begin{array}{l}\mathbf{N} \\
\mathbf{E}\end{array}$ & $\begin{array}{c}\text { EN } \\
\text { E }\end{array}$ & $\begin{array}{l}\mathbf{S} \\
\mathbf{E}\end{array}$ & $\begin{array}{c}\text { ES } \\
\text { E }\end{array}$ & $\mathbf{s}$ & & $\begin{array}{l}\text { FO } \\
\%\end{array}$ \\
\hline Abudefduf saxatilis (Linnaeus, 1758) & & 0 & & c & 0 & & 1 & $x$ & 33,33 \\
\hline Acanthistius brasilianus (Cuvier, 1828) & & 0 & & 1 & 1 & $\mathrm{x}$ & 0 & & 33,33 \\
\hline Acanthostracion quadricornis (Linnaeus, 1758) & Acantrostracion quadricornis & 1 & $\mathrm{x}$ & c & 0 & & 0 & & 33,33 \\
\hline Acanthurus bahianus Castelnau, 1855 & & 1 & $\mathrm{x}$ & c & 0 & & 0 & & 33,33 \\
\hline Acanthurus chirurgus (Bloch, 1787) & & 1 & $\mathrm{x}$ & c & 0 & & 0 & & 33,33 \\
\hline Achirus lineatus (Linnaeus, 1758) & & 1 & & 1 & 1 & & 0 & & 66,67 \\
\hline Membras dissimilis Carvalho, 1956 & Adenops dissimilis & 0 & & c & 0 & & 1 & $x$ & 33,33 \\
\hline Albula vulpes (Linnaeus, 1758) & & 1 & & 1 & 1 & & 1 & & 100,00 \\
\hline Aluterus monoceros (Linnaeus, 1758) & Aluterus monocerus & 1 & $\mathrm{x}$ & c & 0 & & 0 & & 33,33 \\
\hline Anchoa filifera (Fowler, 1915) & & 0 & & 1 & 1 & $\mathrm{x}$ & 0 & & 33,33 \\
\hline Anchoa januaria (Steindachner, 1879) & & 1 & & 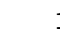 & 1 & & 0 & & 66,67 \\
\hline Anchoa lyolepis Evermann \& Marsh, 1900 & & 1 & & 1 & 1 & & 1 & & 100,00 \\
\hline Anchoa marinii Hildebrand, 1943 & & 1 & & 1 & 1 & & 1 & & 100,00 \\
\hline Anchoa parva (Meek \& Hildebrand, 1943) & & 0 & & c & 0 & & 1 & $\mathrm{x}$ & 33,33 \\
\hline Anchoa spinifer (Valenciennes, 1848) & Anchoa spinifera & 1 & $\mathrm{x}$ & c & 0 & & 0 & & 33,33 \\
\hline Anchoa tricolor (Spix \& Agassiz, 1829) & & 1 & & 1 & 1 & & 1 & & 100,00 \\
\hline Anchovia clupeoides (Swainson, 1839) & & 1 & & 1 & 1 & & 0 & & 66,67 \\
\hline Anchoviella brevirostris (Gunther, 1868) & & 0 & & 1 & 1 & $x$ & 0 & & 33,33 \\
\hline Anchoviella lepidentostole (Fowler, 1911) & & 1 & & 1 & 1 & & 0 & & 66,67 \\
\hline Anisotremus surinamensis (Bloch, 1791) & & 0 & & 1 & 1 & & 1 & & 66,67 \\
\hline Archosargus probatocephalus (Walbaum, 1792) & & 1 & & 1 & 1 & & 0 & & 66,67 \\
\hline Archosargus rhomboidalis (Linnaeus, 1758) & & 1 & & 1 & 1 & & 0 & & 66,67 \\
\hline Aspistor luniscutis (Valenciennes, 1840) & $\begin{array}{c}\text { Arius luniscutis, Sciadeichthys } \\
\text { luniscutis }\end{array}$ & 1 & & 1 & 1 & & 0 & & 66,67 \\
\hline Astroscopus sexspinosus (Steindachner, 1876) & & 0 & & c & 0 & & 1 & $x$ & 33,33 \\
\hline Astroscopus y-graecum (Cuvier, 1829) & Astroscopus ygraecum, Astrocospus & 0 & & 1 & 1 & & 1 & & 66,67 \\
\hline Astyanax bimaculatus (Linnaeus, 1758) & & 0 & & 1 & 1 & $x$ & 0 & & 33,33 \\
\hline Atherinella blackburni (Schultz, 1949) & & 1 & & 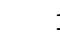 & 1 & & 0 & & 66,67 \\
\hline Atherinella brasiliensis (Quoy \& Gaimard, 1825) & Xenomelaniris brasiliensis & 1 & & 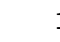 & 1 & & 1 & & 100,00 \\
\hline Bagre bagre (Linnaeus, 1766) & & 0 & & 1 & 1 & $\mathrm{x}$ & 0 & & 33,33 \\
\hline Bagre marinus (Mitchill, 1815) & & 1 & $\mathrm{x}$ & c & 0 & & 0 & & 33,33 \\
\hline Bairdiella ronchus (Cuvier, 1830) & & 1 & $\mathrm{x}$ & c & 0 & & 0 & & 33,33 \\
\hline Bathycongrus dubius (Breder, 1927) & Rhechias dubius & 1 & $\mathrm{x}$ & c & 0 & & 0 & & 33,33 \\
\hline Bathygobius soporator (Valenciennes, 1837) & & 1 & & 1 & 1 & & 0 & & 66,67 \\
\hline Boridia grossidens Cuvier, 1830 & & 1 & & 1 & 1 & & 0 & & 66,67 \\
\hline Bothus ocellatus (Agassiz, 1831) & & 0 & & 1 & 1 & $x$ & 0 & & 33,33 \\
\hline Bothus robinsi Topp \& Roff, 1972 & & 0 & & 1 & 1 & $x$ & 0 & & 33,33 \\
\hline Brevoortia pectinata (Jenyns, 1842) & & 0 & & c & 0 & & 1 & $\mathrm{x}$ & 33,33 \\
\hline Bryx dunckeri (Metzelaar, 1919) & Syngnathus dunkeri & 0 & & 1 & 1 & $x$ & 0 & & 33,33 \\
\hline
\end{tabular}


SANTANA, Fábio M. da S. The ichthyofauna of the brazilian surf zone: a compilation for ecological comprehension per region.

Calamus Penna (Valenciennes, 1830)

Carangoides bartholomaei (Cuvier, 1833)

Caranx crysos (Mitchill, 1815)

Caranx hippos (Linnaeus, 1766)

Caranx latus Agassiz, 1831

Caranx ruber (Bloch, 1793)

Cathorops spixii (Agassiz, 1829)

Centropomus parallelus Poey, 1860

Centropomus undecimalis (Bloch, 1792)

Cetengraulis edentulus (Cuvier, 1829)

Chaetodipterus faber (Broussonet, 1782)

Chaetodon striatus Linnaeus, 1758

Chilomycterus antillarum Jordan \& Rutter, 1897

Chilomycterus spinosus spinosus (Linnaeus, 1758)

Chirocentrodon bleekerianus (Poey, 1867)

Chloroscombrus chrysurus (Linnaeus, 1766)

Citharichthys arenaceus Evermann \& Marsh, 1900

Citharichthys macrops Dresel, 1885

Citharichthys spilopterus Gunther, 1862

Conodon nobilis (Linnaeus, 1758)

Cosmocampus elucens (Poey, 1868)

Cryptotomus roseus Cope, 1871

Ctenogobius boleosoma (Jordan \& Gilbert, 1882)

Ctenogobius stigmaticus (Poey, 1860)

Ctenosciaena gracilicirrhus (Metzelaar, 1919)

Cynoscion leiarchus (Cuvier, 1830)

Cynoscion microlepidotus (Cuvier, 1830)

Cynoscion virescens (Cuvier, 1830)

Dactylopterus volitans (Linnaeus, 1758)

Dactyloscopus crossotus Starks, 1913

Dactyloscopus tridigitatus Gill, 1859

Dasyatis guttata (Bloch \& Scheneider, 1801)

Diapterus auratus Ranzani, 1842

Diapterus rhombeus (Cuvier, 1829)

Diplectrum formosum (Linnaeus, 1766)

Diplectrum radiale (Quoy \& Gaimard, 1824)

Diplodus argenteus argenteus (Valenciennes, 1830)

Discopyge tschudii Heckel, 1846

Dormitator maculatus (Bloch, 1792)

Echeneis naucrates Linnaeus, 1758

Elops saurus Linnaeus, 1766

Engraulis anchoita Hubbs \& Marini, 1935

Epinephelus morio (Valenciennes, 1828)

Etropus crossotus Jordan \& Gilbert, 1882

Eucinostomus argenteus Baird \& Girard, 1855

Eucinostomus gula (Quoy \& Gaimard, 1824)

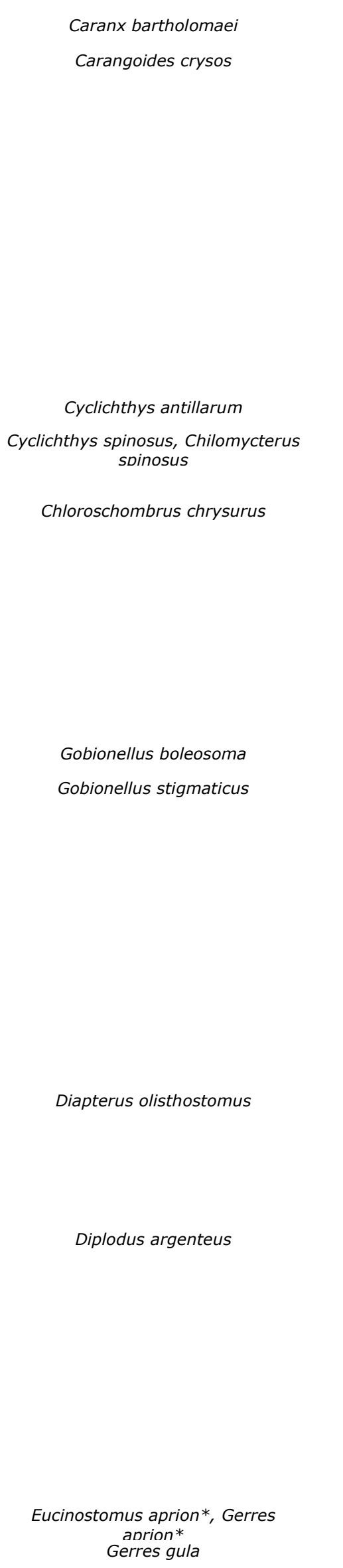

33,33

33,33

66,67

100,00

100,00

33,33

66,67

100,00

100,00

100,00

100,00

33,33

33,33

100,00

100,00

100,00

33,33

100,00

66,67

100,00

100,00

33,33

33,33

66,67

33,33

100,00

66,67

66,67

100,00

33,33

33,33

66,67

33,33

100,00

33,33

33,33

33,33

33,33

33,33

33,33

33,33

66,67

33,33

100,00

100,00

100,00 
SANTANA, Fábio M. da S. The ichthyofauna of the brazilian surf zone: a compilation for ecological comprehension per region.

Eucinostomus lefroyi (Goode, 1874)

Eucinostomus melanopterus (Bleeker, 1863)

Eugerres brasilianus (Cuvier, 1830)

Eugerres lineatus (Humboldt, 1821)

Fistularia petimba Lacepède, 1803

Fistularia tabacaria Linnaeus, 1758

Genidens barbus (Lacepède, 1803)

Genidens genidens (Cuvier, 1829)

Genidens planifrons (Higuchi, Reis \& Araújo, 1982)

Genyatremus luteus (Bloch, 1790)

Gobiesox strumosus Cope, 1870

Gobioides broussonnetti Lacepède, 1800

Gobionellus oceanicus (Pallas, 1770)

Gobionellus stomatus Starks, 1913

Gymnothorax funebris Ranzani, 1839

Gymnotus carapo Linnaeus, 1758

Haemulon aurolineatum Cuvier, 1830

Haemulon bonariense Cuvier, 1830

Haemulon plumierii Lacepède, 1801

Haemulon steindachneri (Jordan \& Gilbert, 1882)

Harengula clupeola (Cuvier, 1829)

Hemiramphus brasiliensis (Linnaeus, 1758)

Heteropriacanthus cruentatus Lacepède, 1801

Hippocampus reidi Ginsburg, 1933

Hyporhamphus roberti roberti (Valenciennes, 1847)

Hyporhamphus unifasciatus (Ranzani, 1841)

Hyporthodus niveatus (Valenciennes, 1828)

Isopisthus parvipinnis (Cuvier, 1830)

Jenkinsia lamprotaenia (Gosse, 1851)

Jenynsia lineata (Jenyns, 1842)

Jenynsia multidentata (Jenyns, 1842)

Kyphosus sectatrix (Linnaeus, 1758)

Labrisomus nuchipinnis (Quoy \& Gaimard, 1824)

Lagocephalus laevigatus (Linnaeus, 1766)

Larimus breviceps Cuvier, 1830

Lile piquitinga (Schreiner \& Miranda Ribeiro, 1903)

Lobotes surinamensis (Bloch, 1790)

Lutjanus analis (Cuvier, 1828)

Lutjanus cyanopterus (Cuvier, 1828)

Lutjanus jocu (Bloch \& Schneider, 1801)

Lutjanus synagris (Linnaeus, 1758)

Lycengraulis grossidens (Agassiz, 1829)

Macrodon ancylodon (Bloch \& Schneider, 1801)

Menticirrhus americanus (Linnaeus, 1758)

Menticirrhus littoralis (Holbrook, 1847)

Microgobius meeki Evermann \& Marsh, 1899

\begin{tabular}{|c|c|c|c|c|c|c|c|}
\hline Ulaema lefroyi, Gerres lefroyi* & 1 & & 1 & & 1 & & 100,00 \\
\hline Gerres melanopterus & 1 & & 1 & & 1 & & 100,00 \\
\hline & 1 & & 1 & & 0 & & 66,67 \\
\hline & 0 & & 1 & $\mathrm{x}$ & 0 & & 33,33 \\
\hline & 1 & & 1 & & 0 & & 66,67 \\
\hline & 1 & & 1 & & 0 & & 66,67 \\
\hline Genidens barba*, Netuma barba & 0 & & 1 & & 1 & & 66,67 \\
\hline & 0 & & 1 & $\mathrm{x}$ & 0 & & 33,33 \\
\hline & 0 & & 0 & & 1 & $\mathrm{x}$ & 33,33 \\
\hline & 1 & $\mathrm{x}$ & 0 & & 0 & & 33,33 \\
\hline & 0 & & 1 & $\mathrm{x}$ & 0 & & 33,33 \\
\hline Gobioides braussonnetti & 0 & & 1 & $\mathrm{x}$ & 0 & & 33,33 \\
\hline & 0 & & 1 & $\mathrm{x}$ & 0 & & 33,33 \\
\hline & 1 & $x$ & 0 & & 0 & & 33,33 \\
\hline & 1 & $\mathrm{x}$ & 0 & & 0 & & 33,33 \\
\hline & 0 & & 1 & $\mathrm{x}$ & 0 & & 33,33 \\
\hline & 1 & $\mathrm{x}$ & 0 & & 0 & & 33,33 \\
\hline Haemulon bonariensis & 1 & $\mathrm{x}$ & 0 & & 0 & & 33,33 \\
\hline Haemulon plumieri & 1 & $\mathrm{x}$ & 0 & & 0 & & 33,33 \\
\hline & 1 & & 1 & & 0 & & 66,67 \\
\hline & 1 & & 1 & & 1 & & 100,00 \\
\hline & 1 & & 1 & & 0 & & 66,67 \\
\hline Priacanthus cruentatus & 0 & & 1 & $\mathrm{x}$ & 0 & & 33,33 \\
\hline Hyppocampus reidi & 1 & & 1 & & 0 & & 66,67 \\
\hline Hyporhamphus roberti & 1 & $\mathrm{x}$ & 0 & & 0 & & 33,33 \\
\hline Hiporhampus unifasciatus & 1 & & 1 & & 1 & & 100,00 \\
\hline Epinephelus niveatus & 0 & & 1 & $\mathrm{x}$ & 0 & & 33,33 \\
\hline & 1 & & 1 & & 1 & & 100,00 \\
\hline & 0 & & 1 & $x$ & 0 & & 33,33 \\
\hline & 0 & & 1 & $\mathrm{x}$ & 0 & & 33,33 \\
\hline & 0 & & 0 & & 1 & $x$ & 33,33 \\
\hline & 1 & $\mathrm{x}$ & 0 & & 0 & & 33,33 \\
\hline & 1 & $\mathrm{x}$ & 0 & & 0 & & 33,33 \\
\hline & 1 & & 1 & & 1 & & 100,00 \\
\hline & 1 & & 1 & & 1 & & 100,00 \\
\hline & 1 & $\mathrm{x}$ & 0 & & 0 & & 33,33 \\
\hline & 1 & & 1 & & 0 & & 66,67 \\
\hline & 1 & $x$ & 0 & & 0 & & 33,33 \\
\hline & 1 & & 1 & & 0 & & 66,67 \\
\hline & 1 & & 1 & & 0 & & 66,67 \\
\hline & 1 & & 1 & & 0 & & 66,67 \\
\hline & 1 & & 1 & & 1 & & 100,00 \\
\hline & 0 & & 1 & $\mathrm{x}$ & 0 & & 33,33 \\
\hline & 1 & & 1 & & 1 & & 100,00 \\
\hline & 1 & & 1 & & 1 & & 100,00 \\
\hline & 1 & $x$ & 0 & & 0 & & 33,33 \\
\hline
\end{tabular}


SANTANA, Fábio M. da S. The ichthyofauna of the brazilian surf zone: a compilation for ecological comprehension per region.

Micropogonias furnieri (Desmarest, 1823)

Monacanthus ciliatus (Mitchill, 1818)

Mugil curema Valenciennes, 1836

Mugil curvidens Valenciennes, 1836

Mugil gaimardianius Desmarest, 1831

Mugil hospes Jordan \& Culver, 1895

Mugil incilis Hancock, 1830

Mugil Liza Valenciennes, 1836

Mugil platanus Gunther, 1880

Mulloidichthys martinicus (Cuvier, 1829)

Mycteroperca bonaci (Poey, 1860)

Myrichthys ocellatus (Lesueur, 1825)

Myrophis punctatus Lutken, 1852

Narcine brasiliensis (Olfers, 1831)

Nicholsina usta usta (Valenciennes, 1840)

Ocyurus chrysurus (Bloch, 1791)

Odontesthes argentinenses (Valenciennes, 1835)

Odontesthes bonariensis (Valenciennes, 1835)

Odontognathus mucronatus Lacepède, 1800

Odontoscion dentex (Cuvier, 1830)

Ogcocephalus vespertilio (Linnaeus, 1758)

Oligoplites palometa (Cuvier, 1832)

Oligoplites saliens (Bloch, 1793)

Oligoplites saurus (Bloch \& Schneider, 1801)

Oncopterus darwinii Steindachner, 1874

Ophioscium punctatissimus Meek \& Hildebrand, 1925

Opisthonema oglinum (Lesueur, 1818)

Orthopristis ruber (Cuvier, 1830)

Paralichthys brasiliensis (Ranzani, 1842)

Paralichthys orbignyanus (Valenciennes, 1839)

Paralonchurus brasiliensis (Steindachner, 1875)

Pareques acuminatus (Bloch \& Schneider, 1801)

Pellona harroweri (Fowler, 1917)

Peprilus paru (Linnaeus,1758)

Percophis brasiliensis Quoy \& Gaimard, 1825

Phalloptychus januarius (Hensel, 1868)

Platanichthys platana (Regan, 1917)

Poecilia vivipara Bloch \& Schneider, 1801

Pogonias cromis (Linnaeus, 1766)

Polydactylus oligodon (Gunther, 1860)

Polydactylus virginicus (Linnaeus, 1758)

Pomacanthus paru (Bloch, 1787)

Pomadasys corvinaeformis (Steindachner, 1868)

Pomadasys crocro (Cuvier, 1830)

Pomadasys ramosus (Poey, 1860)

Pomatomus saltatrix (Linnaeus, 1766)
Pomadasys croco

\begin{tabular}{|c|c|c|c|c|c|c|c|}
\hline & 1 & & 1 & & 1 & & 100,00 \\
\hline & 0 & & 1 & $x$ & 0 & & 33,33 \\
\hline & 1 & & 1 & & 1 & & 100,00 \\
\hline & 1 & $x$ & 0 & & 0 & & 33,33 \\
\hline & 1 & & 1 & & 1 & & 100,00 \\
\hline & 0 & & 0 & & 1 & $x$ & 33,33 \\
\hline & 0 & & 1 & & 1 & & 66,67 \\
\hline & 1 & & 1 & & 1 & & 100,00 \\
\hline & 0 & & 1 & & 1 & & 66,67 \\
\hline & 1 & $x$ & 0 & & 0 & & 33,33 \\
\hline & 0 & & 1 & & 1 & & 66,67 \\
\hline Myrichthys oculatus & 1 & $x$ & 0 & & 0 & & 33,33 \\
\hline & 1 & & 1 & & 0 & & 66,67 \\
\hline & 0 & & 1 & $x$ & 0 & & 33,33 \\
\hline Nicholsina usta & 1 & $x$ & 0 & & 0 & & 33,33 \\
\hline & 1 & & 1 & & 0 & & 66,67 \\
\hline & 0 & & 0 & & 1 & $\mathrm{x}$ & 33,33 \\
\hline & 0 & & 1 & & 1 & & 66,67 \\
\hline & 0 & & 1 & $x$ & 0 & & 33,33 \\
\hline & 1 & $x$ & 0 & & 0 & & 33,33 \\
\hline & 1 & $x$ & 0 & & 0 & & 33,33 \\
\hline & 1 & & 1 & & 0 & & 66,67 \\
\hline & 0 & & 1 & & 1 & & 66,67 \\
\hline & 1 & & 1 & & 1 & & 100,00 \\
\hline & 0 & & 0 & & 1 & $x$ & 33,33 \\
\hline & 1 & & 1 & & 1 & & 100,00 \\
\hline & 1 & & 1 & & 1 & & 100,00 \\
\hline & 1 & & 1 & & 0 & & 66,67 \\
\hline & 1 & & 1 & & 1 & & 100,00 \\
\hline & 0 & & 1 & & 1 & & 66,67 \\
\hline & 0 & & 1 & & 1 & & 66,67 \\
\hline & 1 & $x$ & 0 & & 0 & & 33,33 \\
\hline & 1 & & 1 & & 1 & & 100,00 \\
\hline & 0 & & 0 & & 1 & $X$ & 33,33 \\
\hline & 0 & & 1 & $x$ & 0 & & 33,33 \\
\hline & 0 & & 1 & $x$ & 0 & & 33,33 \\
\hline & 1 & & 1 & & 1 & & 100,00 \\
\hline & 0 & & 1 & $x$ & 0 & & 33,33 \\
\hline & 0 & & 0 & & 1 & $x$ & 33,33 \\
\hline & 0 & & 1 & & 1 & & 66,67 \\
\hline & 1 & & 1 & & 1 & & 100,00 \\
\hline & 0 & & 0 & & 1 & $x$ & 33,33 \\
\hline & 1 & & 1 & & 1 & & 100,00 \\
\hline Pomadasys croco & 0 & & 1 & $x$ & 0 & & 33,33 \\
\hline & 0 & & 0 & & 1 & $\mathrm{x}$ & 33,33 \\
\hline Pomatomus saltator & 0 & & 1 & & 1 & & 66,67 \\
\hline
\end{tabular}


SANTANA, Fábio M. da S. The ichthyofauna of the brazilian surf zone: a compilation for ecological comprehension per region.

Porichthys porosissimus (Cuvier, 1829)

Potamarius grandoculis (Steindachner, 1877)

Hexanematichthys grandoculis

66,67

Prionotus nudigula Ginsburg, 1950

Hexanematichthys grandoculis

Prionotus punctatus (Bloch, 1793)

Pseudocaranx dentex (Bloch \& Schneider, 1801)

Pseudupeneus maculatus (Bloch, 1793)

Rachycentron canadum (Linnaeus, 1766)

Raneya brasiliensis (Kaup, 1856)

Rhinobatos horkelii Muller \& Henli, 1841

Rhinosardinia bahiensis (Steindachner,1879)

Rivulus santensis Kohler, 1906

Rypticus randalli Courtenay, 1967

Salminus brasiliensis (Cuvier, 1816)

Sardinella brasiliensis (Steindachner, 1879)

Scomber japonicus Houttuyn, 1782

Scomberomorus brasiliensis Collette, Russo \&

Zavalla-Camin. 1978

Scorpaena plumieri Bloch, 1789

Selar crumenophthalmus (Bloch, 1793)

Selene setapinnis (Mitchill,1815)

Selene vomer (Linnaeus, 1758)

Seriola rivoliana Valenciennes, 1833

Serranus flaviventris (Cuvier, 1829)

Sparisoma chrysopterum (Bloch \& Schneider, 1801)

Sparisoma radians (Valenciennes, 1840)

Sparisoma rubripinne (Valenciennes, 1840)

Sphoeroides greeleyi Gilbert, 1900

Sphoeroides spengleri (Bloch, 1785)

Sphoeroides testudineus (Linnaeus, 1758)

Sphyraena barracuda (Edwards, 1771)

Sphyraena guachancho Cuvier, 1829

Sphyrhaena tome Fowler, 1903

Scorpaena isthmensis Meek \& Hildebrand, 1928

Stellifer brasiliensis (Schultz, 1945)

Stellifer rastrifer (Jordan, 1889)

Stellifer stellifer (Bloch, 1790)

Stephanolepis hispidus (Linnaeus, 1766)

Strongylura marina (Walbaum, 1792)

Strongylura timucu (Walbaum, 1792)

Rachycentron canadus

Raneya fluminensis

Salminus maxilosus

Sardinella janeiro

Syacium micrurum Ranzani, 1842

Syacium papillosum (Linnaeus, 1758)

Symphurus plagusia (Bloch \& Schneider, 1801) Symphurus papillosum, Scyacium
Dabillosum

Symphurus tesselatus (Quoy \& Gaimard, 1824)

Symphurus trewavasae Chabanaud, 1948

Syngnathus folletti Herald, 1942

Syngnathus pelagicus Linnaeus, 1758

Syngnathus scovelli (Evermann \& Kendall, 1896)

\begin{tabular}{|c|c|c|c|c|c|c|}
\hline & 1 & $x$ & 0 & & 0 & 33,33 \\
\hline & 1 & $x$ & 0 & & 0 & 33,33 \\
\hline & 1 & $x$ & 0 & & 0 & 33,33 \\
\hline & 1 & & 1 & & 1 & 100,00 \\
\hline & 1 & & 1 & & 0 & 66,67 \\
\hline & 1 & & 1 & & 1 & 100,00 \\
\hline & 1 & $x$ & 0 & & 0 & 33,33 \\
\hline & 1 & $\mathrm{x}$ & 0 & & 0 & 33,33 \\
\hline & 0 & & 1 & & 1 & 66,67 \\
\hline & 0 & & 1 & $\mathrm{x}$ & 0 & 33,33 \\
\hline & 0 & & 1 & & 1 & 66,67 \\
\hline & 1 & & 1 & & 1 & 100,00 \\
\hline & 1 & & 1 & & 1 & 100,00 \\
\hline & 1 & & 1 & & 1 & 100,00 \\
\hline & 1 & & 1 & & 1 & 100,00 \\
\hline & 1 & & 1 & & 1 & 100,00 \\
\hline & 0 & & 1 & $x$ & 0 & 33,33 \\
\hline Symphurus papillosum, Scyacium & 0 & & 1 & & 1 & 66,67 \\
\hline & 1 & $\mathrm{x}$ & 0 & & 0 & 33,33 \\
\hline & 1 & & 1 & & 0 & 66,67 \\
\hline & 1 & & 1 & & 0 & 66,67 \\
\hline & 0 & & 1 & & 1 & 66,67 \\
\hline & 1 & $x$ & 0 & & 0 & 33,33 \\
\hline & 1 & & 1 & & 0 & 66,67 \\
\hline
\end{tabular}


SANTANA, Fábio M. da S. The ichthyofauna of the brazilian surf zone: a compilation for ecological comprehension per region.

Synodus foetens (Linnaeus, 1766)

Synodus intermedius (Spix \& Agassiz,1829)

Thalassophryne nattereri Steindachner, 1876

Thyrsitops lepidopoides (Cuvier, 1832)

Trachinocephalus myops (Forster, 1801)

Trachinotus carolinus (Linnaeus, 1766)

Trachinotus falcatus (Linnaeus, 1758)

Trachinotus goodei Jordan \& Evermann, 1896

Trachinotus marginatus Cuvier, 1832

Trichiurus lepturus Linnaeus, 1758

Trinectes microphthalmus (Chabanaud, 1928)

Trinectes paulistanus (Miranda Ribeiro, 1915)

Umbrina canosai Berg, 1895

Umbrina coroides Cuvier, 1830

Upeneus parvus Poey, 1852

Uraspis secunda Wakiya, 1927

Zapteryx brevirostris (Müller \& Henle, 1841)

\begin{tabular}{|c|c|c|c|c|c|c|c|}
\hline & 1 & & 1 & & & & 100,00 \\
\hline & 1 & & 1 & & & & 66,67 \\
\hline \multirow[t]{2}{*}{ Talassophorine nattereri } & 1 & & 1 & & & & 66,67 \\
\hline & 0 & & 1 & $x$ & & & 33,33 \\
\hline \multirow[t]{13}{*}{ Trachynocephalus myops } & 0 & & 1 & $x$ & & & 33,33 \\
\hline & 1 & & 1 & & & & 100,00 \\
\hline & 1 & & 1 & & & & 100,00 \\
\hline & 1 & & 1 & & & & 100,00 \\
\hline & 0 & & 1 & & & & 66,67 \\
\hline & 0 & & 1 & & & & 66,67 \\
\hline & 1 & $x$ & 0 & & & & 33,33 \\
\hline & 1 & & 1 & & & & 66,67 \\
\hline & 0 & & 0 & & & $x$ & 33,33 \\
\hline & 1 & & 1 & & & & 100,00 \\
\hline & 0 & & 1 & $x$ & & & 33,33 \\
\hline & 0 & & 0 & & & 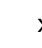 & 33,33 \\
\hline & & & & & $x$ & 0 & $\begin{array}{c}33,3 \\
3\end{array}$ \\
\hline
\end{tabular}

The unique families for each analyzed region are shown in Figure 4.

\section{SOUTHEAST:}

Bothidae, Characidae, Dactyloscopidae, Echeneidae, Elopidae, Gempylidae, Gobiesocidae, Gymnotidae, Narcinidae, Ophidiidae, Percophidae, Poecilidae, Priacanthidae, Rachvcentridae, Rhinobatidae, Rivulidae

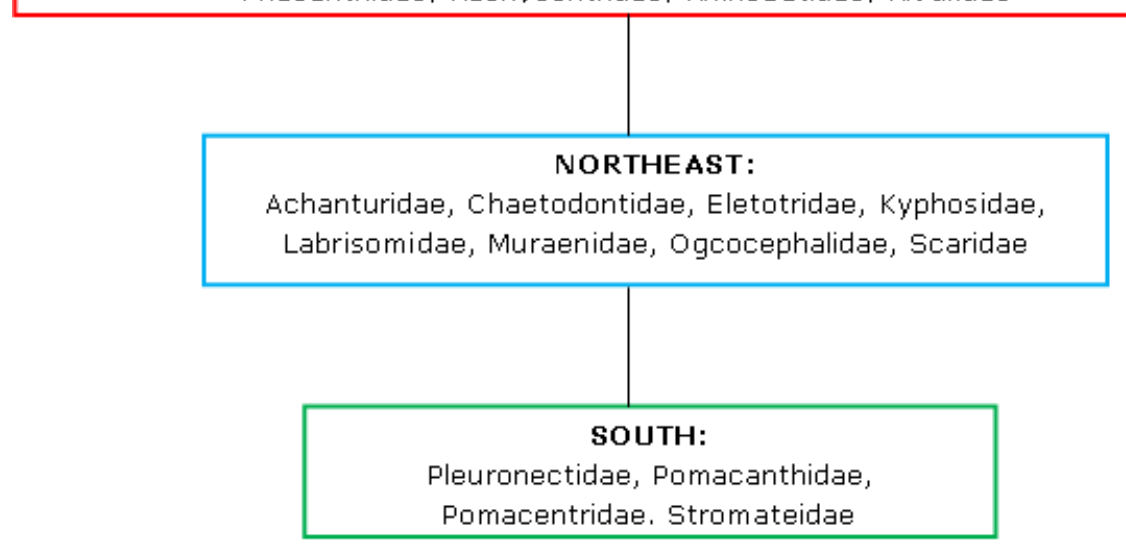

Figure 4 - Fish families recorded exclusively in surf zones of the southeastern, northeastern and southern Brazil.

In the surf zone of the Brazilian beaches, 61 fish species were common to all regions analyzed. The relation of species by territorial region is in Table 2 . The outdated or misspelled scientific names that were cited were validated.

\section{DISCUSSION}

The list of marine ichthyofauna is incomplete and needs further projects and studies, especially in less known areas (MENEZES, 2011). The first step toward an ecological approach and management in a given location is the specific survey (ANDREATTA et al., 2002). Although the Brazilian coast is continental and covers four territorial regions, there were no publications on fish community of surf zones in the northern, and very few to the northeast. Until late 20th 
SANTANA, Fábio M. da S. The ichthyofauna of the brazilian surf zone: a compilation for ecological comprehension per region.

century there were records on the ichthyofauna of these environments in the south and southeast regions. The only exception to the northeast was Teixeira and Almeida (1998). More recently, in the northeast, new researches on this topic have been published: Oliveira-Silva, Peso-Aguiar and Lopes (2008); Lira and Teixeira (2008); Santana and Severi (2009); Gurgel et al. (2012), but it still resents projects that can inventory more consistently its assembly.

All along the Brazilian coast there occur the surf zones - essential for the accomplishment of the life cycle of many marine fish species (PAIVA FILHO and TOSCANO, 1987; GIANNINI and PAIVA FILHO, 1995; GODEFROID et al., 2003; SANTANA and SEVERI, 2009; GURGEL et al., 2012)-. However, the relations of families and species with this environment are not clear yet.

The high primary productivity in the surf zone supports large populations of invertebrates that become visible to predators when the dynamic of the waves moves the sandy substrate (ALLEN and PONDELLA, 2006), attracting many species of fish. For the surf zones, Moyle \& Cech (2000) classified the fish into six categories: small planktivorous and active, substrate wandering tweaks, sole-shaped fish, migratory species, beach spawners and piscivores. The fish that occupy the surf zone are generally small, silvery and planktivorous, including the aterinids, engraulidae, clupeids, which represent the most abundant in these sites. Many representatives of the Scianidae families (e.g. Menticirrhus spp.) are often found using their cirrhi and surrounding the substrate in search of food (ALLEN \& PONDELLA op cite). Concerning Brazil, according to the present survey, these four families were the most specious, besides the Carangidae and Haemulidae.

Two hundred and thirty nine out of the 1,297 species of marine fish described for Brazil (MENEZES, 2011), were listed in this work, considering only the surf zones.

In the northeastern coastal zone there only occurs the seasonal regime of wet and dry seasons (MACEDO, MUNIZ and MONTES, 2004), incurring in a higher incidence of sunny days that are sufficient to increase primary productivity, regardless the season of the year (PASSAVANTE and FEITOSA, 2009). Because of the reduced depth, the shallow waters turn the surf zone into an attractive site for protection and food supply, which boosts the trophic web. Families exclusive to northeast region, in this compilation, are associated with reefs (ARAÚJO and FEITOSA, 2003; HUMAN and DELOACH, 2002), except for Eleotridae, represented by the species Dormitator maculatus. In 2008, Oliveira-Silva et al. recorded the species for surf zones in Bahia beaches, but it was considered estuarine resident by Vasconcelos-Filho et al. (2004). The present results show the existence of connectivity among reef ecosystems and surf zones, featuring the northeastern coast. More than half of the species recorded exclusively for the surf zone of the northeast region were also cited by Paiva et al. (2009) and Araújo and Feitosa (op cite) as associated with reef areas (eg. Gymnotorax funebris, Haemulon aurolineatum, $H$. bonariensis, Lutjanus analis, Sparisoma chrysopterum, S. radians). However, most of them are rare in the surf zones, and use these sites as shelter or attracted by some food supply, as the species Nicholsina usta that uses "Arribadas" algae to the practice of herbivory (SANTANA, 2009). Bairdiella ronchus, species associated with estuarine areas (VASCONCELOS FILHO et al., op cite), was also exclusive of northeastern surf zones. However, unlike the species that are associated with reefs, it is well represented in Cabuçu beach in Bahia (OLIVEIRA-SILVA et al., op cite) and is one of the most frequent and abundant in Jaguaribe beach in Pernambuco (SANTANA and SEVERI, op cite). Bairdiella ronchus usually inhabits the surf zones during the juvenile stage, then migrates to estuaries, where they complete their life cycle (SANTANA et al., in press).

Although studied with greater regularity than in the northeast, most of the works in the south region (8) were in Pontal do Paraná, Paraná state, scarce studies in Rio Grande do Sul (2) and none in Santa Catarina. The missing data could increase the figures about the richness of surf zones in this region. The families Pomacentridae, Pomacanthidae, Stromateidae and Pleuronectidae were exclusive of the surf zone in the south region. The first two are associated with areas of rocky shores (HOSTIM-SILVA et al., 2006). Stromateidae was represented by the Peprilus paru species that occurs throughout the Brazilian coast (FIGUEIREDO and MENEZES, 2000). While in the present survey the species only occurred in this region, Szpilman (2000) highlights that it is rare exactly for the south. Only four species, Abudefduf saxatilis, Caranx 
SANTANA, Fábio M. da S. The ichthyofauna of the brazilian surf zone: a compilation for ecological comprehension per region.

ruber, Pomacanthus paru and Seriola rivoliana of 21 exclusive from surf zone of southern Brazil were cited in the list of species of rocky shores by Hostim-Silva et al. (op cite). Most of the exclusive species are associated with estuaries (eg. Adenops dissimilis, Anchoa parva, Brevoortia pectinata, Pomadasys ramosus) and demersal areas (eg. Pogonias cromis, Ctenosciaena gracilicirrhus, Prionotus nudigula) (FROESE and PAULY, 2012). Two other species, Oncopterus darwinii and Genidens planifrons occur from Santa Catarina down to the coast of Argentina (MENEZES et al., 2003; FROESE and PAULY, op cite), being typical only in this region. As in the south the ichthyofauna suffers influence of the species from Argentina and Uruguay, one would expect more exclusive species in this region, which does not occur. Another hypothesis is that some species, typical from cooler waters have no association with surf zones and use different environment during their life cycle. For being farther from the equator line and closer to the poles, there is a reduction in the decrease of marine diversity (WILLIG et al., 2003), which may be an explanation for this region to show lower number of families and species.

The southeastern surf zones seem to suffer greater influence of the continent, as there were recordings, exclusively, of families whose components are from freshwater (eg. Characidae, Gymnotidae and Rivulidae). Another factor that contributes to higher recording of families in the southeast is the upwelling areas in Cabo Frio, which provide greater primary productivity (BASSANI et al., 1999). The exclusive families in the southeast region compiled in this study do not present association with the reef areas. According to Menezes (2011), the species of rocky bottoms and reef areas are less abundant in Sao Paulo. Studies in this state and in Rio de Janeiro amount more than $90 \%$ of the work compiled. The ichthyofauna of the surf zones of the Southeast is regularly investigated (PAIVA FILHO and TOSCANO, 1987; GIANNINI and PAIVA FILHO, 1995; PESSANHA and ARAÚJO, 2003; GOMES et al., 2003; VASCONCELOS et al., 2007; MONTEIRO-NETO et al., 2008; GONDOLO, MATTOX and CUNNINGHAM, 2011), contributing to a greater record of families and species. As the study and the sample period are extended, more rare species are being recorded (ENGEN, 2007), even if in small quantities. Among the typical freshwater fish found exclusively in the southeast region, Poecilia vivipara and Rivulus santensis were recorded by Giannini \& Paiva Filho (op cite) for the coast of São Paulo state. Neither migrates and both are from freshwater environment (FROESE and PAULY, op cite), but the former has already been recorded for estuarine area by Paiva et al. (2009). Salminus brasiliensis and Gymnotus carapo are potamodromous (Froese \& Pauly, op cite) and occurred in Gargaú beach, which is adjacent to the Itabapoama river, located in the state of Rio de Janeiro (GOMES et al., op cite). Probably, they must have been taken to the surf zone by some flood. Astyanax bimaculatus occurred in Itamambuca beach, in São Paulo state (GONDOLO et al., op cite) and although not characteristic of the surf zone, it has already been recorded for the estuary (PAIVA et al., op cite).

\section{CONCLUSIONS}

Finally, the presence of 239 fish species and 71 families represent a considerable figure for the ichthyofauna of the surf zone of the Brazilian coast, because this environment is still scarcely studied. However, because we can find species that inhabit other environments and for the presence of 61 species with $100 \%$ frequency of occurrence in the three regions studied, it can be concluded that it is an ecologically important site. It is hoped that with increased effort and more studies in the northeastern and southern states, and beginning the survey in the north region, this list might increase considerably turning the ecological importance of these areas more valorized.

\section{ACKNOLEDGEMENTS}

To Apolônio Salles Educational Development Foundation (Fundação Apolônio Salles de Desenvolvimento Educacional- FADURPE), for the logistical support essential to the accomplishment of this work. To The Support to Science Foundation of Pernambuco State (Fundação de Amparo a Ciência do estado de Pernambuco - FACEPE) and CNPq, for granting the $\mathrm{PhD}$ scholarship to the former author, and researcher scholarship to the latter author. To Biologist Anailza Cristina, for the invaluable collaboration during the collection activities. 
SANTANA, Fábio M. da S. The ichthyofauna of the brazilian surf zone: a compilation for ecological comprehension per region.

\section{REFERENCES}

AB'SÁBER, A. N. Litoral do Brasil. Metalivros, São Paulo, 2003. 281 p.

ALLEN, L. G.; PONDELLA, D. J. II. 2006. Surf Zone, Coastal Pelagic Zone, and Harbors. In: The Ecology of Marine Fishes: California and Adjacent Waters (ALLEN, L. G.; PONDELLA, D. J. II; HORN, M. (ed.). University of California Press, Los Angeles, 2006, p. 149 - 166.

ANDREATA, J. V.; MAURER, B. C.; BAPTISTA, M. G. S.; MANZANO, F. V.; TEIXEIRA, D. E.; LONGO, M. M.; FERRET, N. V. Composição da assembléia de peixes da Baía da Ribeira, Angra dos Reis, Rio de Janeiro, Brasil. Revista Brasileira de Zoologia, v. 19, n. 4, p. 1139-1146, 2002.

ARAÚJO, M. E. FEITOSA, C.V. Análise de agrupamento da ictiofauna recifal do Brasil com base em dados secundários: uma avaliação crítica. Tropical Oceanography, v. 31, n. 2, p. 181-201, 2003.

ARAÚJO, M. E. TEIXEIRA, J. M. C. \& OliVEIRA, A.M.E. Peixes estuarinos marinhos do nordeste brasileiro: Guia Ilustrado. Editora UFC, Fortaleza, 2004. 260 p.

ARAÚJO, C. C. V.; ROSA, D. M.; FERNANDES, J. M.; RIPOLI, L. V.; KROHLING, W. Composição e estrutura da comunidade de peixes de uma praia arenosa da Ilha do Frade, Vitória, Espírito Santo. Iheringia, v. 1, n. 98, p. 129-135, 2008.

BARLETTA, M.; BLABER, S. J. M. Comparision of fish assemblages and guilds in tropical habitats of the Embley (Indo-West Pacific) and Caeté (Western Atlantic) estuaries. Bulletin of Marine Science, v. 80, n. 3, p. 647-680, 2007.

BASSANI, C.; BONECKER, A. C. T.; BONECKER, S. L. C.; NOGUEIRA, C. R.; REIS, J. M. L.;

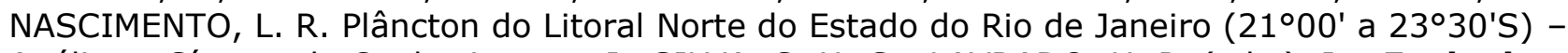
Análise e Síntese do Conhecimento. In SILVA, S. H. G.; LAVRADO, H. P. (eds.). In: Ecologia dos Ambientes Costeiros do Estado do Rio de Janeiro. Série Oecologia Brasiliensis. Vol. 7. Rio de Janeiro: PPGECO/UFRJ, 1999. p. 99-120.

BLABER, S. J. M.; BLABER, T. J. Factors affecting the distribution of juvenile estuarine and inshore fish. Journal of Fish Biology, v. 17, p.143-162, 1980.

ENGEN, S. Heterogeneous communities with lognormal species abundance distribution: Species-area curves and sustainability. Journal of Theoretical Biology, v. 249, p. 791-803, 2007.

FÉLIX, F. C.; SPACH, H. L.; MORO, O. S.; SCHWARZ, J. R.; SANTOS, C.; HACKRADT, C. W.; HOSTIM, M. S. Utilization patterns of surf zone inhabiting fish from beaches in Southern Brazil. Pan-American Journal of Aquatic Sciences, v. 2, n. 1, p. 27-39, 2007(a).

FÉLIX, F. C.; SPACH, H. L.; MORO, O. S.; HACKRADT, C. W.; QUEIROZ, G. M. L. N.; HOSTIMSILVA, M. Ichthyofauna composition across a wave-energy gradient on Southern Brazil beaches. Brazilian Journal of Oceanography, v. 55, n. 4, p. 281-292, 2007(b).

FÉLIX-HACKRADT, F. C.; SPACH, H. L.; MORO, P. S.; PICHLER, H. A.; MAGGI, A. S.; HOSTIMSILVA, M.; HACKRADT, C. W. Diel and tidal variation in surf zone fish assemblages of a sheltered beach in southern Brazil. Latinoamerican Journal of Aquatic Research, v. 38, n. 3, p. 447-460, 2010.

Figueiredo, J. L.; MENEZeS, N.A. Manual de Peixes Marinhos do Sudeste do Brasil. VI. Teleostei (5). Museu de Zoologia, Universidade de São Paulo, São Paulo, 2000. 116 p.

FROESE, R.; PAULY, D. Eds. FishBase. World Wide Web electronic

publication. Disponível em: http://www.fishbase.org acessado em 01 de Nov.2012.

GAELZER, L. R.; MACHADO, G. R.; BAPTISTA, O. R.; ZALMON, I. R. Surf-Zone Ichthyofauna Diel Variation in Arraial do Cabo, Southeastern Brazil. Journal of Coastal Research, Special Issue 39, 2006. 
SANTANA, Fábio M. da S. The ichthyofauna of the brazilian surf zone: a compilation for ecological comprehension per region.

GIANNINI, R.; PAIVA FILHO, A. M. Análise comparativa da ictiofauna da zona de arrebentação de praias arenosas do estado de São Paulo, Brasil. Boletim do Instituto Oceanográfico, v. 43, n. 2, p. 141-152, 1995.

GODEFROID, R. S.; HOFSTAETTER M.; SPACH H. L. Moon, tidal and diel influences on catch composition of fishes in the surf zone of Pontal do Sul beach, Paraná. Revista Brasileira de Zoologia, v. 15, n. 3, p. 647-701, 1998.

GODEFROID, R. S.; SANTOS, C.; HOFSTAETTER, M.; SPACH, H. L. Occurrence of Larvae and Juveniles of Eucinostomus argenteus, Eucinostomus gula, Menticirrhus americanus, Menticirrhus littoralis, Umbrina coroides and Micropogonias furnieri at Pontal do Sul beach, Paraná. Brazilian Archives of Biology and Technology, v. 44, n. 4, p. 411-418, 2001.

GODEFROID, R. S.; SPACH, H. L.; SCHWARZ, R. J.; QUEIROZ, M. G. A fauna de peixes da praia do Balneário Atami, Paraná, Brasil. Atlântica, v. 25, n. 2, p. 147-161, 2003.

GOMES, M. P.; CUNHA, M. S.; ZALMON, I. L. 2003. Spatial and temporal variations of diurnal ichthyofauna on surf-zone of São Francisco do Itabapoana beaches, Rio de Janeiro state, Brazil. Brazilian Archives of Biology and Technology, v. 46, n. 4, p. 653-664, 2003.

GONDOLO, G. F.; MATTOX, G. M. T.; CUNNINGHAM, P. T. M. Ecological aspects of the surf-zone ichthyofauna of Itamambuca Beach, Ubatuba, SP. Biota Neotropica, v. 11, n. 2, p. 183-192, 2011.

GURGEL, T. A. B., OLIVEIRA, M. R., BRASIL, D. F., CHELLAPPA, S. Peixes marinhos das águas costeira da Ponta Negra, Rio Grande do Norte, Brasil. Biota Amazônia, Macapá, v. 2, n. 1, p. 83-97, 2012.

HOSTIM-SILVA, M.; ANDRADE, A. B.; MACHADO, L. F.; GERHARDINGER, L. C.; DAROS, F. A.; BARREIROS, J. P.; GODOY, E. A. S. Peixes de costão rochoso de Santa Catarina:

Arvoredo. Itajaí, Universidade do Vale do Itajaí, 2006. 134 p.

HUMANN, P.; DELOACH, N. Reef Fish Identification: Florida Caribbean Bahamas. New World Publications, Inc., 2002. 481 p.

KNOPPERS, B.; EKAU, W.; FIGUEIREDO JR, E. A. G.; SOARES-GOMES, A. Zona costeira e plataforma continental do Brasil. In: PEREIRA, R. C.; SOARES-GOMES, A. Biologia Marinha. Rio deJaneiro, Interciência, 2002. p. 353-360.

LASIAK, T. A. Structural aspects of the surf-zone fish assemblage at King's beach Algoa Bay, South Africa: long-term fluctuations. Estuarine, Coastal and Shelf Science, v. 18, p. 459483, 1984(a).

LASIAK, T. A. Structural aspects of the surf zone fish assemblage at King's Beach, Algoa Bay, South Africa: Short - term fluctuations. Estuarine, Coastal and Shelf Science, v. 18, n. 4, p. 347-360, 1984(b).

LIMA, M. S. P.; VIEIRA, J. P. Variação espaço-temporal da ictiofauna da zona de arrebentação da Praia do Cassino, Rio Grande do Sul, Brasil. Zoologia, v. 26, n. 3, p. 499-510, 2009.

LIRA, A. K. F.; TEIXEIRA, S. F. Ictiofauna da Praia de Jaguaribe, Itamaracá, Pernambuco.

Iheringia, Zoologia, v. 98, n. 4, p. 785-780, 2008.

MACÊDO, S. J.; MUNIZ, K.; MONTES, M. J. F. Hidrologia da região costeira e plataforma continental do estado de Pernambuco. In: ESKINAZI LEÇA, E.; NEUMANN LEITÃO, S.; COSTA, M. F. (orgs.). Oceanografia: um cenário tropical. Recife, Bagaço. 2004. p. 255-286.

MENEZeS, N. A., BUCKUP, P. A., FIGUeIREDO, J. L.; MOURA, R. L. Catálogo das espécies de peixes marinhos do Brasil. Museu de Zoologia, Universidade de São Paulo, São Paulo, 2003. $159 \mathrm{p}$.

MENEZES, N. A. Checklist dos peixes marinhos do Estado de São Paulo, Brasil. Biota

Neotropica, v. 11, Supl.1, p. 33-46, 2011. 
SANTANA, Fábio M. da S. The ichthyofauna of the brazilian surf zone: a compilation for ecological comprehension per region.

MONT'ALVERNE, R.; MORAES, L. E.; RODRIGUES, F. L.; VIEIRA, J. P. Do mud deposition events on sandy beaches affect surf zone ichthyofauna? A southern Brazilian case study. Estuarine Coastal and Shelf Science, v. 102-103, n. 2012, p. 116-125, 2012.

MONTEIRO-NETO, C.; TUBino, R. A.; MORAES, L. E. S.; NETO, J. P. M.; ESTEVES, G. V.; FORTES, W. L. Associações de peixes na região costeira de Itaipu, Niterói, RJ. Iheringia, Zoologia, v. 98, n. 1, p. 50-59, 2008.

MOYLE, P. B.; CECH, J. J. Fishes: an Introduction to Ichthyology. 4 ed. Upper Saddle River, Prentice Hall, 2000. 726 p.

OLIVEIRA-SILVA, J. T.; PESO-AGUIAR, M. C.; LOPES, P. R. Ictiofauna das praias de Cabuçu e Berlinque: Uma contribuição ao conhecimento das comunidades de peixes na Baía de Todos os Santos, Bahia, Brasil. Biotemas, v. 21, n. 4, p. 105-115, 2008.

PAIVA A. C. G.; LIMA M. F. V.; SOUZA, J. R. B.; ARAUJO, M. E. Spatial distribution of the estuarine ichthyofauna of the Rio Formoso (Pernambuco, Brazil), with emphasis on reef fish. Zoologia, v. 26, p. 266-278, 2009.

PAIVA FILHO, A. M.; TOSCANO, A. P. Estudo comparativo e variação sazonal da ictiofauna na zona entremarés do Mar Casado-Guarujá e Mar Pequeno-São Vicente, SP. Boletim do Instituto Oceanográfico, v. 35, p. 153-165, 1987.

PASSAVANTE, J. Z. O.; FEITOSA, F. A. N. Dinâmica da produtividade fitoplanctônica na zona costeira marinha. In: ESKINAZI LEÇA, E.; NEUMANN LEITÃO, S.; COSTA, M. F. (orgs.).

Oceanografia: um cenário tropical. Recife, Bagaço. 2004. p. 425-439.

PASSOS, A. C. 2012. Ictiofauna do complexo estuarino de Paranaguá e áreas adjacentes. Curitiba, 2012. 118f. Dissertação (Mestrado em Sistemas Costeiros e Oceânicos). Universidade Federal do Paraná.

PESSANHA, A. L. M.; ARAÚJO, F. G. Spatial, temporal and diel variations of fish assemblages at two sandy beaches in the Sepetiba Bay, Rio de Janeiro, Brazil. Estuarine Coastal and Shelf Science, v. 57, p. 1-12, 2003.

PRIMER-E. Plymouth Routines in Multivariate Ecological Research. Plymouth: Plymouth Marine Laboratory, 2000.

ROBERTSON, A. I.; LENANTON, R. C. J. Fish community structure and food chain dynamics in the surf-zone of sandy beaches: the role of detached macrophyte detritus. Journal of Experimental Marine Biology and Ecology, v. 84, p. 265-283, 1984.

SANTANA, F. M. S. Ictiofauna da zona de arrebentação da praia de Jaguaribe, Itamaracá, Pernambuco: composição, abundância e distribuição mensal. Recife, 2009. 97f. Dissertação (Mestrado em Recursos Pesqueiros e Aquicultura) - Departamento de Pesca e Aquicultura. Universidade Federal Rural de Pernambuco.

SANTANA, F. M. S.; SEVERI, W. Composição e estrutura da assembléia de peixes da zona de arrebentação da praia de Jaguaribe, Itamaracá - Pernambuco. Bioikos, v. 23, n. 1, p. 3-17, 2009.

SANTANA, F. M. S; SEVERI, W.; ÁRAÚJO, M. E. The influence of seasonality on fish early life stages and residence in surf zones: a case study in a tropical region. Biota Neotropica (No prelo).

SZPILMAN, M. Peixes marinhos do Brasil: guia prático de identificação. Aqualittera e Mauad Editora, RJ, 2000. 288 p.

TEIXEIRA, R. L.; ALMEIDA, G. I. Composição da ictiofauna de três praias arenosas de MaceióAL, Brasil. Boletim do Museu de Biologia Mello Leitão, v. 8, p. 21-38, 1998.

VASCONCELLOS, R. M.; SANTOS, J. N. S.; SILVA, M. A.; ARAúJO, F. G. Efeito do grau de exposição às ondas sobre a comunidade de peixes juvenis em praias arenosas do Município do Rio de Janeiro, Brasil. Biota Neotropica, v. 7, n. 1, p. 171-178, 2007. 
SANTANA, Fábio M. da S. The ichthyofauna of the brazilian surf zone: a compilation for ecological comprehension per region.

VASCONCELOS FILHO, A.; GUEDES, D. N.; TEIXEIRA, S. F.; OlIVEIRA, A. M. E. Peixes marinhos costeiros e estuarinos. In: ESKINAZI LEÇA, E.; NEUMANN LEITÃO, S.; COSTA, M. F. (orgs.). Oceanografia: um cenário tropical. Recife, Bagaço, 2004. p. 555-570.

VELOSO, V. G.; NEVES, G. Praias arenosas. In: Pereira, R.C.; SOARES-GOMES, A. (Eds). Biologia Marinha, Rio de Janeiro, Interciência, 2009. p. 339-360.

WHITFIELD, A. K. A review of estuarine ichthyology in South Africa over the past 50 years. Transactions of the Royal Society of South Africa, v. 51, p. 79-89, 1996.

WILLIG, M. R.; KAUFMAN, D. M.; STEVENS, R. D. Latitudinal gradients of biodiversity: Patterns, processes, and synthesis. Annual Review of Ecology, Evolution, and Systematics, v. 34, p. 273-309, 2003.

WRIGHT, L. D.; SHORT A. D. Morphodynamics of Beaches and Surf Zones in Australia. In: Komar, P. D. (ed). Handbook of Coastal Process and Erosion. CRC Press, Boca Raton, 1984. p 35-66. 